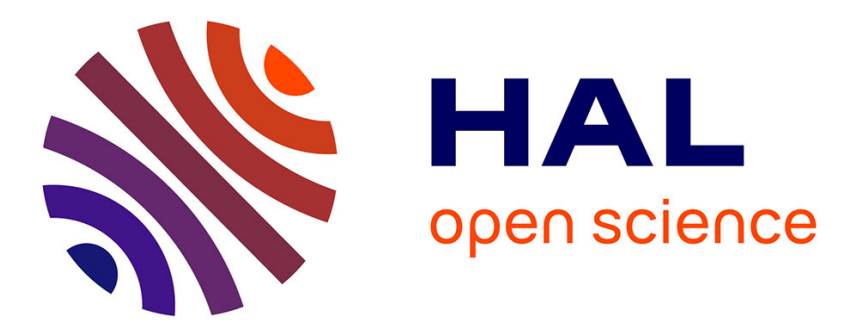

\title{
ÉTUDE EXPÉRIMENTALE DU RALENTISSEMENT DANS LES GAZ DE L'ÉJECTION DE MATIÈRE INDUITE PAR CHOC
}

\author{
C. Remiot, P. Elias, P. Chapron, M. Mondot
}

\section{To cite this version:}

C. Remiot, P. Elias, P. Chapron, M. Mondot. ÉTUDE EXPÉRIMENTALE DU RALENTISSEMENT DANS LES GAZ DE L'ÉJECTION DE MATIÈRE INDUITE PAR CHOC. Journal de Physique IV Proceedings, 1991, 01 (C3), pp.C3-447-C3-452. 10.1051/jp4:1991363 . jpa-00250507

\section{HAL Id: jpa-00250507 https://hal.science/jpa-00250507}

Submitted on 1 Jan 1991

HAL is a multi-disciplinary open access archive for the deposit and dissemination of scientific research documents, whether they are published or not. The documents may come from teaching and research institutions in France or abroad, or from public or private research centers.
L'archive ouverte pluridisciplinaire HAL, est destinée au dépôt et à la diffusion de documents scientifiques de niveau recherche, publiés ou non, émanant des établissements d'enseignement et de recherche français ou étrangers, des laboratoires publics ou privés. 


\begin{abstract}
Résumé - Nous décrivons une méthode expérimentale utilisant simultanément la radiographie $X$-éclair et la caméra ultra-rapide à image intégrale en ombroscopie dans le but d'étudier le ralentissement de l'éjection de matière induite par choc dans un gaz dense situé en avant de la surface libre. Les expériences ont été réalisées avec de l'étain choqué à $40 \mathrm{GPa}$ par un générateur explosif d'onde plane ou à $16 \mathrm{GPa}$ avec un générateur explosif à relèvement latéral et à différents gaz (argon, azote, hélium) à différentes pressions initiales (de 0 à $0,8 \mathrm{MPa}$ ). Avec l'argon, nous notons un ralentissement important des particules éjectées et quelquefois un arrêt (quelques microsecondes après le débouché du choc dans le gaz), qui dépend directem: nt de la pression initiale de l'argon. L'azote, entraîne un ralentissement moins important que l'argon et l'hélium, n'a aucune influence sur le déplacement des particules éjectées.

Abstract - We describe an experimental technique using both flash X-Ray radiograrhy and ultra-high speed framing camera shadowgraphy in order to study the slowing duwn of shock-induced matter ejection appearing when a dense gaz is put ahead the sample free surface. Experiments have been achieved with tin shocked at $40 \mathrm{GPa}$ by an explosive plane wave generator or at $16 \mathrm{GPa}$ by an explosive lateral wave generator and different gas (argon, nitrogen, helium) at different initial pressures (from 0 to $0.8 \mathrm{MPa}$ ). With argon gas, we show up a drastic slowing down of the ejected particles and, sometimes, a quick stopping (a few microseconds after shock bursts into the gas), which directly depends on the argon initial pressure. Nitrogen induces a slowing down less strong than argon, and helium has no influence on the ejected particle motion.
\end{abstract}

\title{
1. INTRODUCTION
}

Il a été montré qu'après la réflexion d'une onde de choc sur la surface libre d'un échantillon métallique, un nuage de particules est éjecté en avant de la surface libre. La vitesse et la masse totale éjectée dépendent de nombreux paramètres tels que la nature de l'échantillon, l'aspect géométrique de la surface libre et l'intensité du choc incident $/ 1 / 2 / / 3 /$.

Dans ce papier, nous étudions la vitesse d'expansion de ces particules en avant de la surface libre en présence d'un gaz. Les expériences ont été réalisées avec de l'étain pour lequel la quantité de matière éjectée et la vitesse d'éjection sous choc sont bien connues /4/, et avec différents gaz à des pressions initiales comprises entre 0 et $0,8 \mathrm{MPa}$.

\section{MONTAGE EXPERIMENTAL ET DIAGNOSTIC}

Les montages expérimentaux sont constitués d'une enceinte permettant de maintenir le gaz sous pression, de deux hublots autorisant les observations par ombroscopie optique et radiographie $\mathrm{X}$ éclair. Des traversées électriques étanches sont utilisées pour le passage des fils des sondes de chronométrie. L'échantillon d'étain de $3 \mathrm{~mm}$ d'épaisseur ainsi qu'une plaque d'acier de $1,5 \mathrm{~mm}$ d'épaisseur ferment hermétiquement la chambre haute pression. La surface de l'échäntillon d'étain est pour moitié polie optique et pour l'autre usinée avec des rainures parallèles de profondeur $100 \mu \mathrm{m}$ et de période $300 \mu \mathrm{m}$ dans le cas du choc frontal (figure 1) ou uniquement usinée des mêmes rainures dans le cas du choc latéral (figure 2). 


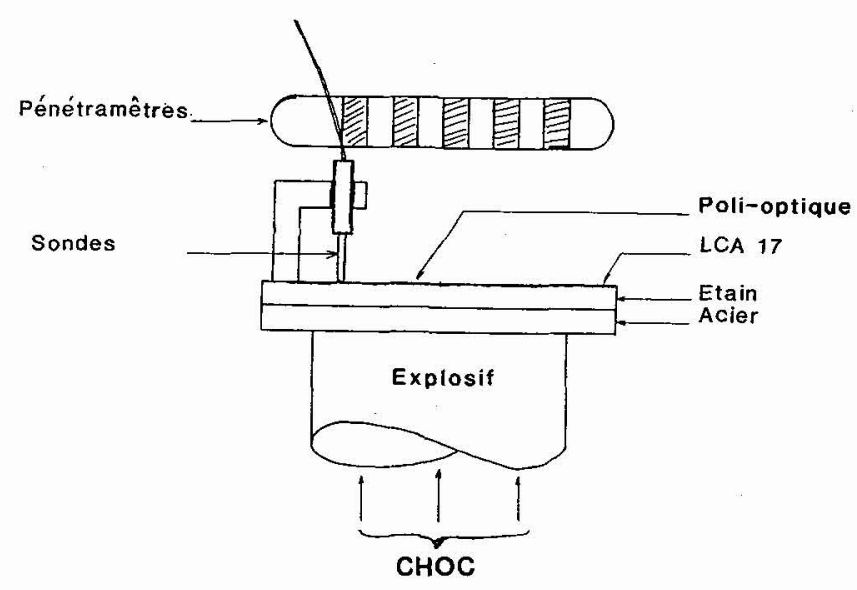

FIGURE 1 : Montage choc frontal

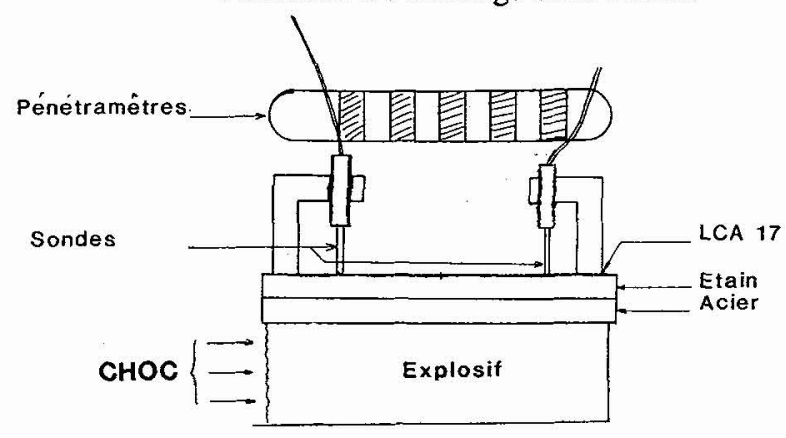

FIGURE 2 : Montage choc latéral

Deux diagnostics complémentaires sont utilisés conjointement dans le but d'étudier le déplacement de l'ejecta dans le gaz : l'ombroscopie optique et la radiographie X éclair /5//6/.

Nous réalisons l'observation optique par l'utilisation d'une caméra Barr \& Stroud type CP5 en fonction images intégrales avec un temps interimage de $139 \mathrm{~ns}$, soit un temps de pause de $50 \mathrm{~ns}$. La prise de vue en ombroscopie est obtenue au moyen d'un flash électronique qui sert à éclairer l'arrière du phénomène à étudier. La radiographie $X$ éclair est obtenue à l'aide d'un générateur Scandiflash 600 donnant, pour une tension de $600 \mathrm{kV}$, une dose de $20 \mathrm{mrad}$ à $1 \mathrm{~m}$ pendant $20 \mathrm{~ns}$, la tache focale étant de l'ordre de $1,2 \mathrm{~mm}$ de diamètre. L'ensemble expérimental est présenté sur la figure 3.

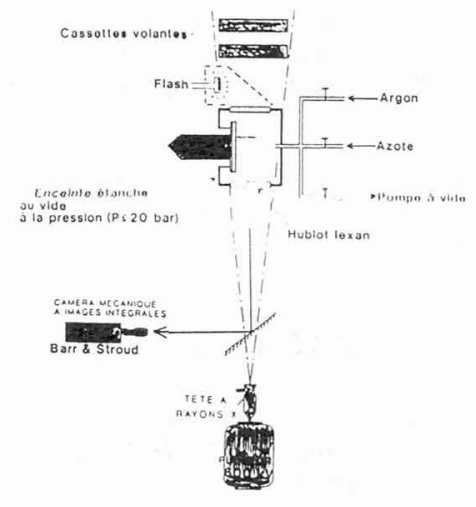

FIGURE 3 : Dispositif expérimental 
Nous observons par ombroscopie optique l'évolution du front d'éjection dans le gaz en fonction du temps. Par radiographie X éclair, la répartition de l'ejecta et la distance du front d'éjection à la surface libre sont obtenues à un instant donné pour les expériences sous choc frontal, alors que pour le relèvement latéral, nous observons directement l'évolution au cours du temps de la position du front d'éjection par rapport à la surface libre. La masse volumique moyenne dans l'ejecta est obtenue par comparaison entre la densité optique de l'ejecta et celle obtenue par les pénétramètres. La mesure de la densité optique est réalisée à l'aide d'un microdensitomètre Joyce \& Loebl couplé à un Apple II.

\section{EXPERIENCES ET RESULTATS}

\subsection{Choc frontal.}

Six expériences ont été réalisées en utilisant trois gaz différents (argon, azote, hẻlium) pour des pressions initiales de gaz de 0 à $0,8 \mathrm{MPa}$. Le tableau 1 donne les conditions initiales des expériences. L'instant zéro correspond à l'instant de la réflexion du choc en surface libre de l'étain qui est détecté par 2 sondes de chronométrie.

\begin{tabular}{|c|c|c|c|cc|c|}
\hline $\begin{array}{c}\text { Pression } \\
\text { de choc } \\
\text { dans } \\
\text { l'étain } \\
(\mathrm{GPa})\end{array}$ & $\begin{array}{c}\text { Nature } \\
\text { du gaz }\end{array}$ & $\begin{array}{c}\text { Pression } \\
\text { initiale } \\
\mathrm{du} g a z \\
(\mathrm{MPa})\end{array}$ & $\begin{array}{c}\text { Rayon X } \\
\text { Instant } \\
\text { du flash } \\
(\mu \mathrm{s})\end{array}$ & $\begin{array}{c}|c| \\
\text { Ombroscopie } \\
\text { Temps } \\
\text { interimage } \\
(\mathrm{ns})\end{array}$ & $\begin{array}{c}\text { Nombre } \\
\text { d'images }\end{array}$ & $\begin{array}{c}\text { Distance } \\
\text { d'arrêt (L) } \\
(\mathrm{mm})\end{array}$ \\
\hline 40 & Vide & 0 & 3,425 & 139 & 117 & pas d'arrêt \\
40 & Argon & 0,15 & 3,334 & 139 & 117 & $\begin{array}{c}\text { pas d'arrêt } \\
\text { avant } 7 \text { us } \\
40\end{array}$ \\
40 & Argon & 0,4 & 3,572 & 139 & 117 & 3,4 \\
40 & Argon & 0,8 & 3,449 & 139 & 117 & 2,4 \\
40 & Azote & 0,8 & 3,980 & 139 & 117 & 5,0 \\
\end{tabular}

TABLEAU 1

La figure 4 montre les radiographies obtenues avec l'argon pour des pressions initiales de 0 à $0,8 \mathrm{MPa}$ et la figure 5 présente les radiographies obtenues pour une pression de $0,8 \mathrm{MPa}$ de 3 différents gaz (argon, azote, hélium). Pour chaque expérience, nous obtenons une différentielle entre la surface polie optique et la surface usinée. Nous observons une diminution d'épaisseur de la zone d'ejecta lorsque la pression initiale de gaz augmente et lorsque le numéro atomique du gaz augmente.

Par ombroscopie, nous mesurons un ralentissement de la tête d'éjection qui dépend directement de la pression initiale de gaz (figure 6) et de la nature du gaz (figure 7). Nous notons un bon accord entre les deux diagnostics. Nous définissons un paramètre $L$ appelé distance d'arrêt $/ 7 /$, qui correspond à la distance maximale entre la surface libre et la tête d'éjection. 


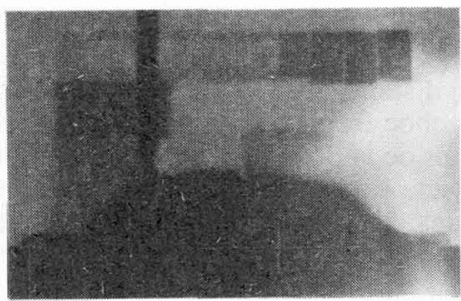

VIDE $T=4,78 \mu \mathrm{s}$

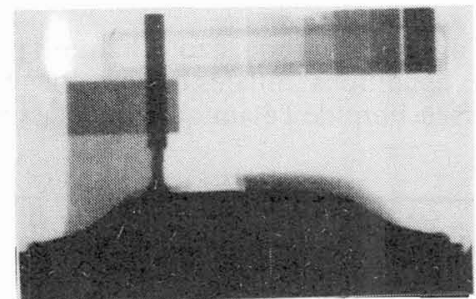

$0,4 \mathrm{MPa} \mathrm{T}=3,57 \mu$ s $\underline{\text { FIGURE } 4}$

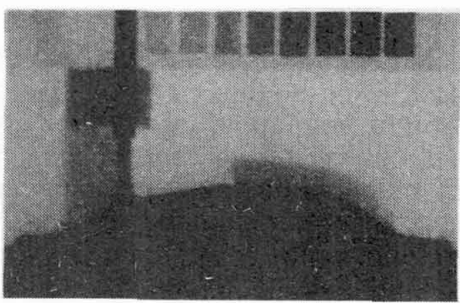

$0,15 \mathrm{MPa} \quad \mathrm{T}=3,33 \mu \mathrm{s}$

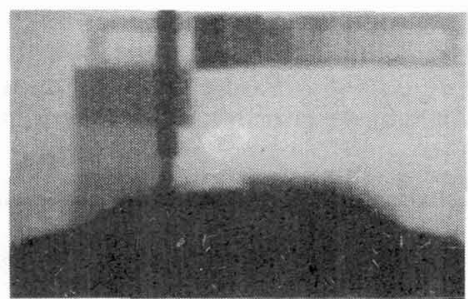

$0,8 \mathrm{MPa} \quad \mathrm{T}=3,45 \mu \mathrm{s}$
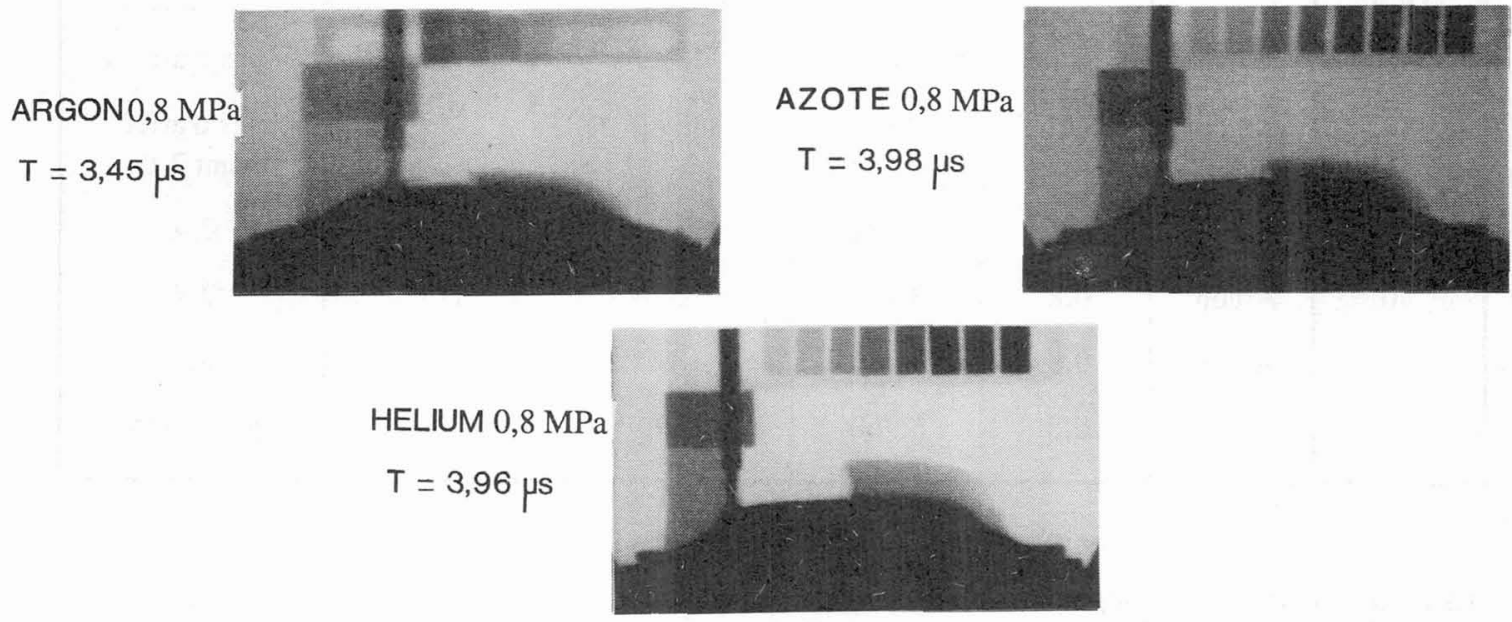

FIGURE 5

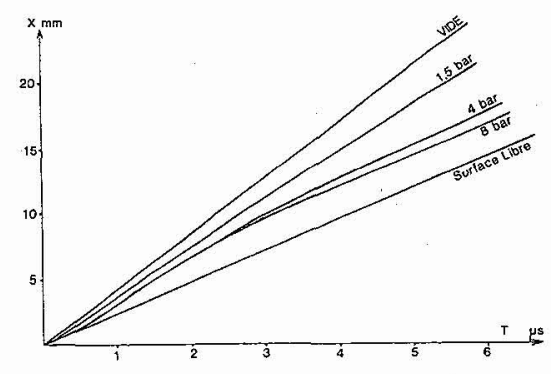

FIGURE 6

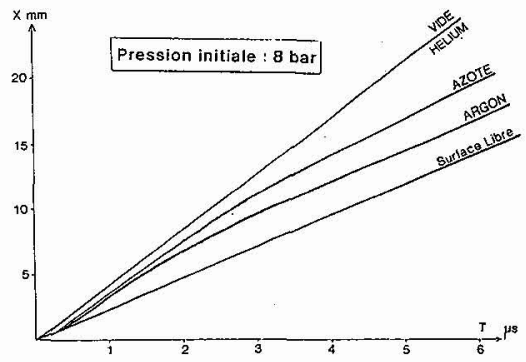

FIGURE 7 


\subsection{Choc latéral.}

Trois expériences ont été réalisées en utilisant deux gaz (argon et hélium) à des pressions initiales différentes $(0,4$ et $0,8 \mathrm{MPa})$. Le tableau 2 donne les conditions initiales des expériences.

\begin{tabular}{|c|c|c|c|cc|}
\hline $\begin{array}{c}\text { Pression } \\
\text { de choc } \\
\text { dans } \\
\text { l'étain } \\
(\mathrm{GPa})\end{array}$ & $\begin{array}{c}\text { Nature } \\
\text { du gaz }\end{array}$ & $\begin{array}{c}\text { Pression } \\
\text { initiale } \\
\text { du gaz } \\
(\mathrm{MPa})\end{array}$ & $\begin{array}{c}\text { Rayon X } \\
\text { Instant } \\
\text { du flash } \\
(\mu \mathrm{s})\end{array}$ & $\begin{array}{c}\text { Temps } \\
\text { interimage } \\
(\mathrm{ns})\end{array}$ & $\begin{array}{c}\text { Nombre } \\
\text { d'images }\end{array}$ \\
\hline 16 & Argon & 0,4 & 8,3 & 139 & 117 \\
16 & Argon & 0,8 & 8,3 & 139 & 117 \\
16 & Hélium & 0,8 & 8,3 & 139 & 117 \\
\hline
\end{tabular}

TABLEAU 2

La figure 8 montre les radiographies obtenues. Nous observons directement le ralentissement de la tête d'éjection et nous connaissons en chaque point la distance entre la surface libre et la tête d'éjection.

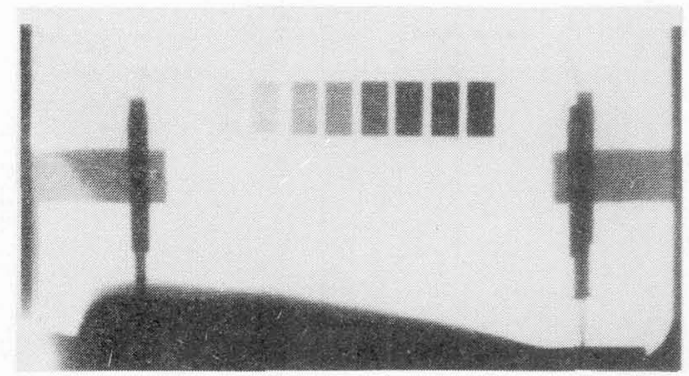

Argon $0,8 \mathrm{MPa}$

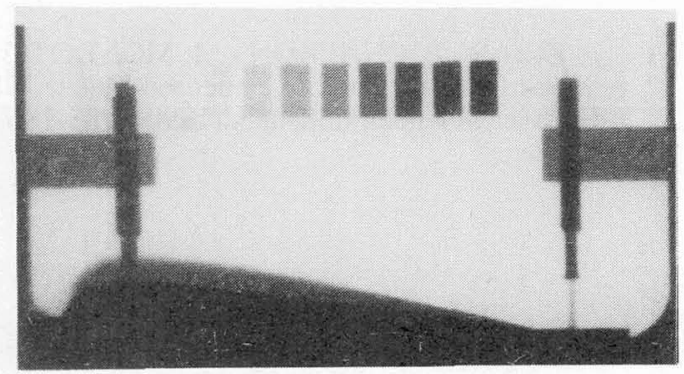

Argon $0,4 \mathrm{MPa}$

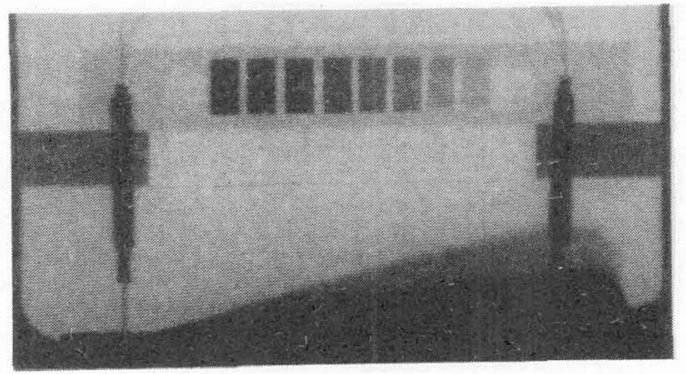

Hélium $0,8 \mathrm{MPa}$

FIGURE 8

\section{CONCLUSION}

Les expériences réalisées montrent une bonne homogénéité des résultats obtenus par deux techniques différentes : l'ombroscopie et la radiographie $X$ éclair. Nous avons pu définir une distance d'arrêt des particules et montrer qu'elle dépend fortement de la nature du gaz et de son numéro atomique. En effet, plus le gaz a un nombre atomique élevé plus la distance d'arrêt est faible, il en est de même lorsque l'on augmente la pression initiale du gaz. 


\section{REFERENCES}

/1/ J.R. ASAY - L.P. MIX - F.C. PERRY Applied Physics Letters.

Vol. $29, \mathrm{n}^{\circ} 5$ (September 1976), 284-287.

21 R. CHERET - P. CHAPRON - P. ELIAS - J. MARTINEAU Shock Waves in Condensed Matter 1985, ed. Y. Gupta. Plenum (1986), 651-654.

B/ R. COUCH - L. SHAW - R. BARLETT - L. STEINMETZ - W. BEHRENDT - C. FIRPO Journal de Physique.

Supplément au $\mathrm{n}^{\circ} 8$, Tome 46 (1985), C5-385, DYMAT 85.

14/ V. FRACHET - P. ELIAS - J. MARTINEAU

Shock Waves in Condensed Matter 1987, eds. S.C. Schmidt and N.C. Holmes. Elsevier Science Publishers (1988), 235-238.

15/ C. BIZEUIL - P. CHAPRON - P. ELIAS - V. FRACHET - B. LAURENT Journal de Physique.

Supplément au n 8 , Tome 46 (1985) C5-351, DYMAT 85.

16/ P. ELIAS - P. CHAPRON

Shock Waves in Condensed Matter 1985, ed. Y. Gupta.

Plenum (1985), 645-50

III P. ELIAS - P. CHAPRON - M. MONDOT

Shock Compression of Condensed Matter 1989.

Elsevier Science Publishers (1990), 783-786. 